\title{
JORDAN TRIPLE FORMS OF JORDAN ALGEBRAS
}

\author{
ERHARD NEHER ${ }^{1}$
}

\begin{abstract}
We show that a central nondegenerate Jordan triple system over a field of characteristic $\neq 2$ has a nonzero center iff it is scalar isomorphic to a Jordan algebra. As an application we classify Jordan triple forms of Jordan algebras.
\end{abstract}

Throughout we are working with Jordan triple systems over a field $k$ of characteristic $\neq 2$. The reason for this restriction is that there seems to be no good definition for the center of a Jordan triple system or Jordan algebra in characteristic 2. For the first half of this note we do not assume finite dimensionality.

For a Jordan triple system $V$ with left multiplication $L$ the center of $V$ is defined as the subspace

$$
C(V)=\{c \in V ; L(c, v)=L(v, c) \text { for all } v \in V\} .
$$

The reason for this notation is made clear by the following

EXAMPLE. Let $g$ be a Jordan algebra. By forgetting the squaring operation or the unit element, if $g$ has one, we obtain a Jordan triple system denoted by $V(\mathcal{f})$. The left multiplication $R$ of $g$ and $L$ of $V(g)$ are connected in the following way:

$$
L(x, y)=2[R(x), R(y)]+2 R(x y) .
$$

This formula implies that the center of $\mathscr{g}$ and of $V(\mathcal{g})$ coincide.

Some fundamental properties of the center are put together in the following

LEMMA 1. (a) Let $c_{1}, c_{2} \in C(V)$ and $u, v \in V$ arbitrary. Then

$$
\left[L\left(c_{1}, c_{2}\right), L(u, v)\right]=0=\left[L\left(c_{1}, c_{2}\right), P(u, v)\right] .
$$

(b) $C(V)$ is a subsystem of $V$.

(c) If $K$ is an extension field of $k$, then $K \otimes C(V)=C(K \otimes V)$.

Proof. (a) and (b) are proved in [3] and (c) is shown as usual, since the conditions on $C(V)$ are linear.

We need the notion of "scalar isomorphism" invented by J. Faulkner and J. Ferrar. The author is indebted to N. Hopkins who introduced him to this concept. One calls two Jordan triple systems $V_{1}$ and $V_{2}$ scalar isomorphic if there exists a vector space isomorphism $\varphi: V_{1} \rightarrow V_{2}$ and a $\zeta \in k^{*}$ (called the multiplier) such that $\varphi\{x y z\}_{1}=\zeta\{\varphi x, \varphi y, \varphi z\}_{2}$ for all $x, y, z \in V_{1}$.

Received by the editors May 20, 1982

1980 Mathematics Subject Classification. Primary 17C20.

Key words and phrases. Jordan triple systems, center, forms of Jordan algebras.

'Partially supported by Deutsche Forschungsgemeinschaft. 
REMARKS. (1) Scalar isomorphism is an equivalence relation.

(2) A scalar isomorphism maps ideals onto ideals. In particular, it preserves simpleness.

(3) Let $\varphi$ be a scalar isomorphism with multiplier $\zeta$. Then $\xi \varphi, \xi \in k^{*}$, is a scalar isomorphism with multiplier $\xi^{-2} \zeta$. This shows that multipliers occurring with scalar isomorphisms are only determined up to a square. In particular, if $k$ is algebraically closed, "scalar isomorphic" is equivalent to "isomorphic".

(4) A scalar isomorphism maps $C\left(V_{1}\right)$ onto $C\left(V_{2}\right)$. In particular, every Jordan triple system scalar isomorphic to a unital Jordan algebra has a nonzero center. To prove a partial converse of this statement we need the following criterion:

LEMMA 2. Let $V$ be a Jordan triple system with quadratic representation $P$. Then $V$ is scalar isomorphic to a unital Jordan algebra iff there exists $e \in V$ and $\zeta \in k^{*}$ with $P(e)=\zeta \mathrm{Id}$.

Proof. If $\varphi: q \rightarrow V$ is a scalar isomorphism between a unital Jordan algebra $q$ and $V$ with multiplier $\zeta$, then $P\left(\varphi e_{q}\right)=\zeta^{-1}$ Id where $e_{g}$ is the unit of $g$. Conversely, $\tilde{P}(x):=\zeta^{-1} P(x)$ defines on $V$ a Jordan triple structure $\tilde{V}$ which obviously is scalar isomorphic to $V$. Because $\tilde{P}(e)=\mathrm{Id}, \tilde{V}$ is the Jordan triple associated to a unital Jordan algebra [2, X, Theorem 1].

We also need the notion of the centeroid of $V$ which is the centralizer of $\{L(u, v) ; u, v \in V\} \cup\{P(u, v) ; u, v \in V\}$ in $\operatorname{End}_{k} V$. It is denoted by $Z(V)$ and contains $k$ Id. One calls $V$ central, if $Z(V)=k$ Id.

THEOREM 1. Let $V$ be central and nondegenerate (i.e. $P(x)=0$ implies $x=0$ ). Then $V$ has a nonzero center iff $V$ is scalar isomorphic to a unital Jordan algebra.

Proof. By Remark (4) we only have to consider the case when $V$ has a nonzero center. We choose $0 \neq c \in C(V)$. Then $L(c, c)=2 P(c) \in Z(V)$ by Lemma 1 (a). Therefore $P(c)=\zeta$ Id for some $\zeta \in k^{*}$ by centralness and nondegeneracy. Now Lemma 2 implies the claim.

The assumption of Theorem 1 can be achieved for the class of simple finitedimensional Jordan triple systems. For one proves as for algebras that in this case the centroid $Z(V)$ is an extension field of $k$ Id and $V$ viewed as a triple system over $Z(V)$ is central-simple. Obviously, the center of $V$ over $k$ and of $V$ over $Z(V)$ coincide. Thus it is no harm to assume central-simplicity right away. Since simpleness and finite dimensionality imply nondegeneracy we obtain

COROLLARY 1. Let $V$ be a finite-dimensional central-simple Jordan triple system over $k$. Then $C(V) \neq 0$ iff $V$ is scalar-isomorphic to a unital central-simple Jordan algebra over $k$.

We will apply Corollary 1 to the problem of classifying Jordan triple forms of Jordan algebras. So we are given a Jordan algebra $g$ over an extension field $K$ of $k$ and we look for triple systems $V$ over $k$ which are subtriple systems of $V(q)$ over $k$ and satisfy $K \otimes V=V(g)$. There are obvious candidates for such forms: We take a Jordan algebra $k$-form $\mathbb{Q}$ of $g$ and $\zeta \in k^{*}$. If $U$ denotes the quadratic representation 
of $Q$, then $U_{\zeta}(x)=\zeta U(x)$ defines on $\mathbb{Q}$ the structure of a Jordan triple $k$-form of $g$ which is denoted by $V(Q, \zeta)$. The following theorem says that these are all Jordan triple forms of $g$.

THEOREM 2. Let $g$ be a central-simple finite-dimensional Jordan algebra over $K$ and $k$ a subfield of $K$. Let $\mathbf{E}$ be a representative system for the set of all isomorphy classes of Jordan algebra $k$-forms of $g$ and $\mathbf{D}$ a representative system for the group $k^{*} / k^{* 2}$. Then every Jordan triple $k$-form of $\mathcal{G}$ is isomorphic to exactly one $V(\mathbb{Q}, \zeta)$ with $Q \in \mathbf{E}$, $\zeta \in \mathbf{D}$.

Proof. Let $V$ be a Jordan triple $k$-form of $g$. Then $V$ is a central-simple since $V(g)$ is, and Lemma $1(c)$ shows $C(V) \neq 0$ because $g$ is unital. Now Corollary 1 and the proof of Lemma 2 imply $V=V(\tilde{Q}, \tilde{\zeta})$ for a Jordan algebra $k$-form $\mathbb{Q}$ of $g$ and $\zeta \in k^{*}$. Since $\zeta$ is determined up to a square we obtain that $V$ is isomorphic to some $V(\mathbb{Q}, \zeta), \mathbb{Q} \in \mathbf{E}, \zeta \in \mathbf{D}$.

It remains to prove that if $\varphi: V(\mathbb{Q}, \zeta) \rightarrow V(\mathscr{B}, \xi)$ is a Jordan triple isomorphism then $Q \simeq \mathscr{B}$ and $\zeta k^{* 2}=\xi k^{* 2}$. We immediately get $V(\mathbb{Q}, 1) \simeq V\left(\mathscr{B}, \zeta^{-1} \xi\right)$, so $\mathbb{Q} \simeq \mathscr{B}$ as soon as $\zeta^{-1} \xi$ is a square. But since $U\left(\varphi e_{\mathscr{Q}}\right)=\zeta \xi^{-1} \mathrm{Id}, e_{\mathscr{Q}}=$ unit element of $\mathscr{Q}$, and $\mathscr{B}$ is central, the assertion is a consequence of the following lemma whose proof is omitted since it is a trivial generalisation of [1, IV, Satz 2.5].

LEMMA 3. Let $\mathscr{B}$ be a unital Jordan algebra over $k$ and $x \in \mathscr{B}$. Then $U(x)=\alpha$ Id for some $\alpha \in k$ iff $x \in C(q)$ and $x^{2}=\alpha e_{4 g}$.

\section{REFERENCES}

1. H. Braun and M. Koecher, Jordan-Algebren, Die Grundlehren der Math. Wissenschaften, Band 128 , Springer-Verlag, Berlin, Heidelberg and New York, 1965.

2. K. Meyberg, Lectures on algebras and triple systems, Lecture Notes, University of Virginia, Charlottesville, 1972.

3. E. Neher, Jordan triple systems with completely reducible derivation or structure algebras, preprint 1982.

MATHEMATISChes INSTITUT DER WeSTFALISCHE WILHELMS-UNIVERSITAT, EINSTEINSTR. 64/IV, 4400 MUNSTER, WEST GERMANY 